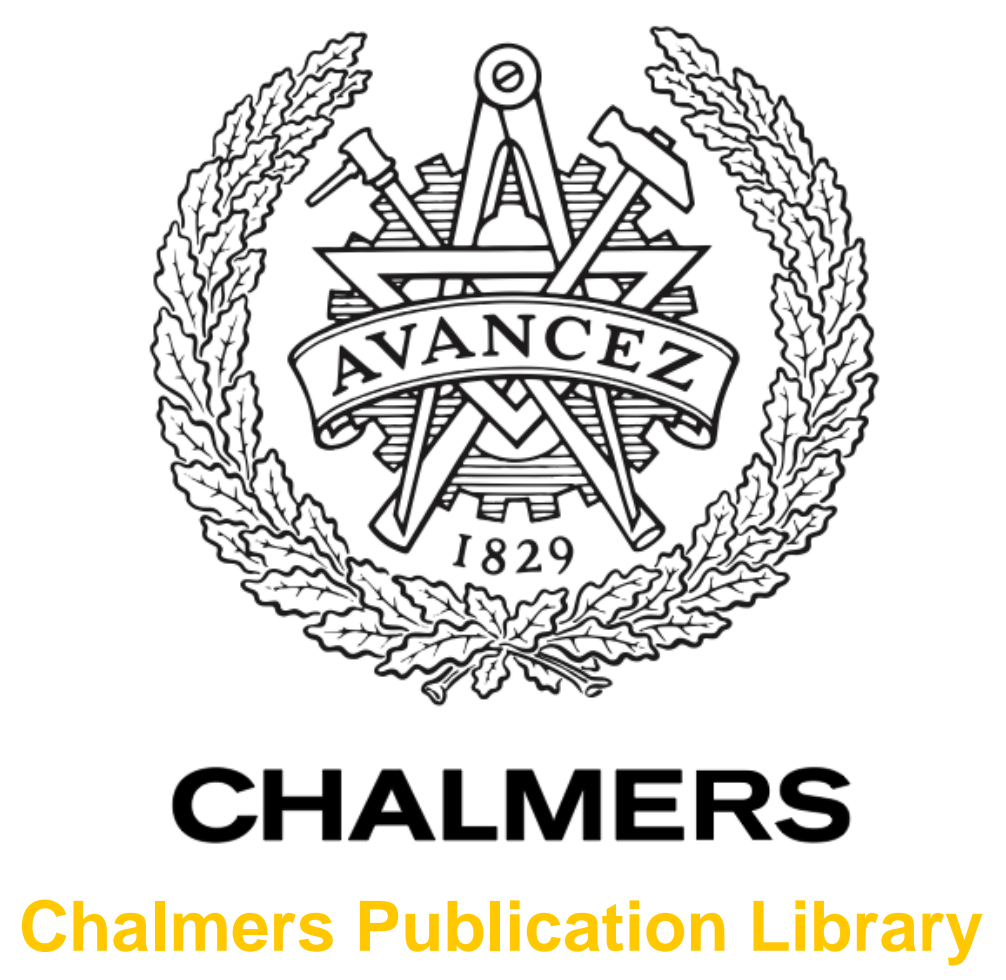

On the sum rate of $\mathrm{ZF}$ detectors in correlated $\mathrm{K}$ fading MIMO channels

This document has been downloaded from Chalmers Publication Library (CPL). It is the author's version of a work that was accepted for publication in:

in Proc. IEEE International Conference on Acoustics, Speech and Signal Processing (ICASSP)

Citation for the published paper:

Matthaiou, M. ; Chatzidiamantis, N. ; Karagiannidis, G. (2011) "On the sum rate of ZF detectors in correlated $\mathrm{K}$ fading MIMO channels". in Proc. IEEE International Conference on Acoustics, Speech and Signal Processing (ICASSP)

Downloaded from: http://publications.lib.chalmers.se/publication/135710

Notice: Changes introduced as a result of publishing processes such as copy-editing and formatting may not be reflected in this document. For a definitive version of this work, please refer to the published source. Please note that access to the published version might require a subscription.

Chalmers Publication Library (CPL) offers the possibility of retrieving research publications produced at Chalmers University of Technology. It covers all types of publications: articles, dissertations, licentiate theses, masters theses, conference papers, reports etc. Since 2006 it is the official tool for Chalmers official publication statistics. To ensure that Chalmers research results are disseminated as widely as possible, an Open Access Policy has been adopted.

The CPL service is administrated and maintained by Chalmers Library. 


\title{
ON THE SUM RATE OF ZF DETECTORS OVER CORRELATED $K$ FADING MIMO CHANNELS
}

\author{
Michail Matthaiou*, Nestor D. Chatzidiamantis ${ }^{\dagger}$, and George K. Karagiannidis ${ }^{\dagger}$ \\ * Department of Signals and Systems, Chalmers University of Technology, Gothenburg, Sweden \\ ${ }^{\dagger}$ Department of Electrical and Computer Engineering, Aristotle University of Thessaloniki, Greece \\ Email: michail.matthaiou@chalmers.se,\{nestoras, geokarag\}@auth.gr
}

\begin{abstract}
This paper presents a detailed sum rate investigation of Zero-Forcing (ZF) detectors over composite multiple-input multiple-output (MIMO) channels. To this end, we consider the generic $K$ distribution (Rayleigh/gamma distribution) to model the composite fading fluctuations and also assume the general case of semi-correlated small-scale fading. Novel exact analytical expressions are derived for the achievable sum rate followed by asymptotic expressions in the low Signalto-Noise ratio (SNR) regime. In parallel, new, closed-form upper and lower bounds on the sum rate are derived that remain tight for all SNRs. The theoretical analysis is validated via a set of Monte-Carlo simulations.
\end{abstract}

\section{INTRODUCTION}

The capacity of point-to-point MIMO communication systems has been well investigated under Rayleigh fading conditions and assuming all different types of spatial correlation $[1,2]$. However, little is still known for the capacity of distributed MIMO (D-MIMO) systems which exploit both spatial micro and macro-diversity [3-5]. The main feature of these systems is that multiple antennas at one end of the wireless channel are packed into multiple radio ports that are spatially separated. Hence, each link experiences different degree of path loss, as a result of the different access distances, along with different shadowing effects. Note that the latter manifestation is rather critical when assessing MIMO performance since it can significantly diminish the benefits of MIMO technology.

In this paper, we elaborate on the performance of ZF linear receivers over such composite D-MIMO channels. The fading fluctuations are modeled via the $K$-distribution $[6,7]$ which is a generic model that occurs when small-scale fading is modeled via the Rayleigh distribution and large-scale fading via the gamma distribution. This model has been demonstrated to effectively approximate most of the fading and shadowing effects occurring in wireless channels, and also to be analytically friendlier than the Rayleigh/lognormal model. Besides, this important implication was recently re- ported in [8], where the authors examined the achievable sum rate of ZF detectors over Rayleigh/lognormal MIMO channels. The final result, though, is given in integral form (see [8, Eq. (15)]) which was numerically approximated via Gauss-Hermite polynomials. This technique, however, is time-consuming (especially at low SNRs) and not amenable to further manipulations. In addition, the presented analysis was tied to co-located MIMO antenna arrays.

We hereafter pursue a statistical analysis of ZF detectors over composite $K$ MIMO channels and also consider the more general case of correlated Rayleigh fading with correlation on the side with the minimum number of antennas. The exact achievable sum rate is analytically derived followed by a tractable affine expansion in the asymptotically low-SNR regime. In addition, we propose novel, closed-form upper and lower bounds on the sum rate. These expressions yield useful insights into the implications of the model parameters on the ZF detector's performance.

Notation: We use upper and lower case boldface to denote matrices and vectors, respectively. The $n \times n$ identity matrix reads as $\mathbf{I}_{n}$. The expectation is given by $\mathbb{E}[\cdot]$ while the symbols $(\cdot)^{\dagger}$ and $(\cdot)^{H}$ represent the pseudo-inverse and Hermitian transpose of a matrix, respectively.

\section{MIMO SYSTEM MODEL}

Let us consider a typical D-MIMO system equipped with $N_{r}$ receive antennas and $L$ radio ports each connected to $N_{t}$ transmit antennas and also require that $L N_{t} \leq N_{r}$. In the following, we assume that the transmitter equally splits the available average power, $P$, amongst all data streams. Then, the input-output relationship reads as

$$
\mathbf{y}=\sqrt{\frac{P}{L N_{t}}} \mathbf{H} \boldsymbol{\Xi}^{1 / 2} \mathbf{s}+\mathbf{n}
$$

where $\mathbf{y} \in \mathbb{C}^{N_{r} \times 1}$ is the received signal vector, $\mathbf{s} \in \mathbb{C}^{L N_{t} \times 1}$ is the vector containing the transmitted symbols which are drawn from a unit-power constellation while the complex AWGN term is zero-mean with covariance $\mathbb{E}\left[\mathbf{n n}^{H}\right]=$ $N_{0} \mathbf{I}_{N_{r}}$. The diagonal matrix $\Xi \in \mathbb{R}^{L N_{t} \times L N_{t}}$ represents the 
large-scale fading, and hence $\boldsymbol{\Xi}=\operatorname{diag}\left\{\mathbf{I}_{N_{t}} \xi_{m} / D_{m}^{v}\right\}_{m=1}^{L}$ where $D_{m}$ denotes the distance between the receiver and the $m$-th radio port while $v$ is the path-loss exponent. The largescale fading coefficients $\xi_{m}, m=1, \ldots, L$, are modeled as gamma random variables (RVs), or

$$
f_{\xi_{m}}\left(\xi_{m}\right)=\frac{\xi_{m}^{k_{m}-1}}{\Gamma\left(k_{m}\right) \Omega_{m}^{k_{m}}} \exp \left(-\frac{\xi_{m}}{\Omega_{m}}\right), \xi_{m}, \Omega_{m}, k_{m} \geq 0
$$

where $k_{i}, \Omega_{i}=\mathbb{E}\left[\xi_{i}\right] / k_{i}$, are the so-called shape and scale parameters of the gamma distribution respectively while $\Gamma(\cdot)$ is the well-known gamma function. As was previously mentioned, the small-scale fading is assumed to follow a semicorrelated Rayleigh distribution and as such, we can express $\mathbf{H} \in \mathbb{C}^{N_{r} \times L N_{t}}$ according to $\mathbf{H}=\mathbf{H}_{w} \mathbf{R}_{t}^{1 / 2}$ where the entries of $\mathbf{H}_{w}$ are modeled as i.i.d. $\mathcal{C N}(0,1) \mathrm{RVs}$ while $\mathbf{R}_{t}$ is the positive definite covariance matrix of every row of $\mathbf{H}$.

After defining $\mathbf{Z} \triangleq \mathbf{H} \boldsymbol{\Xi}^{1 / 2}$, the $\mathrm{ZF}$ filter matrix becomes $\mathbf{G}=\left(P / L N_{t}\right)^{-1 / 2} \mathbf{Z}^{\dagger}[9]$. Assuming independent decoding, the received signal is decomposed into $L N_{t}$ parallel streams with the instantaneous received SNR at the $m$-th ZF output $\left(1 \leq m \leq L N_{t}\right)$ being

$$
\gamma_{m} \triangleq \frac{\rho}{L N_{t}\left[\left(\mathbf{Z}^{H} \mathbf{Z}\right)^{-1}\right]_{m m}}=\frac{\rho[\boldsymbol{\Xi}]_{m m}}{L N_{t}\left[\left(\mathbf{H}^{H} \mathbf{H}\right)^{-1}\right]_{m m}}
$$

where $\rho=P / N_{0}$ is the average SNR and $[\cdot]_{m m}$ returns the $m$-th diagonal element of a matrix. We can also define the random small-scale counterpart as follows

$$
x_{m}=\frac{1}{\left[\left(\mathbf{H}^{H} \mathbf{H}\right)^{-1}\right]_{m m}}
$$

which follows a complex semi-correlated central Wishart distribution $x_{m} \sim \mathcal{C W}\left(N_{r}-L N_{t}+1,1 /\left[\mathbf{R}_{t}^{-1}\right]_{m m}\right)$ and its PDF was explicitly given in [10, Theorem 1]

$$
f_{x_{m}}\left(x_{m}\right)=\frac{\sigma_{m} e^{-x_{m} \sigma_{m}}}{\left(N_{r}-L N_{t}\right) !}\left(x_{m} \sigma_{m}\right)^{N_{r}-L N_{t}}
$$

where $\sigma_{m}$ is the $m$-th diagonal entry of $\mathbf{R}_{t}^{-1}$.

\section{ACHIEVABLE SUM RATE OF ZF DETECTORS}

\subsection{Exact analysis}

The maximum achievable sum rate, assuming independent decoding at the receiver, is essentially the summation of the throughputs offered by each subchannel, or

$$
R \triangleq \sum_{m=1}^{L N_{t}} \mathbb{E}\left[\log _{2}\left(1+\gamma_{m}\right)\right]=\sum_{m=1}^{L N_{t}} \mathbb{E}\left[\log _{2}\left(1+\frac{\rho \xi_{m} x_{m}}{L N_{t} D_{m}^{v}}\right)\right]
$$

where the expectation is taken over all realizations of $\mathbf{H}$ and $\Xi$. The following theorem returns an analytical expression for the exact sum rate of $\mathrm{ZF}$ detectors:
Theorem 1 The achievable sum rate of $Z F$ detectors over correlated K MIMO channels is given by

$$
\begin{aligned}
R & =\frac{1}{\ln 2\left(N_{r}-L N_{t}\right) !} \sum_{m=1}^{L N_{t}} \frac{1}{\Gamma\left(k_{m}\right)} \\
& \times G_{4,2}^{1,4}\left[\frac{\rho \Omega_{m}}{L N_{t} \sigma_{m} D_{m}^{v}} \mid \begin{array}{c}
\left.1-k_{m}, L N_{t}-N_{r}, 1,1\right] \\
1,0
\end{array}\right]
\end{aligned}
$$

where $G_{p, q}^{m, n}\left[x, \mid \begin{array}{c}\alpha_{1}, \ldots, \alpha_{p} \\ \beta_{1}, \ldots, \beta_{q}\end{array}\right]$ is the Meijer's G-function [11, Eq. (9.301)].

Proof: The proof starts by expressing the expectation in (6) in integral form as

$$
\begin{aligned}
R=\frac{1}{\ln 2} \sum_{m=1}^{L N_{t}} & \int_{0}^{\infty} \int_{0}^{\infty} \ln \left(1+\frac{\rho \xi_{m} x_{m}}{L N_{t} D_{m}^{v}}\right) \\
& \times f_{\xi_{m}}\left(\xi_{m}\right) f_{x_{m}}\left(x_{m}\right) d \xi_{m} d x_{m} .
\end{aligned}
$$

Substituting (2) and (5) into (8) and successively applying [11, Eq. (7.813.1)], we can obtain (7) after some basic algebraic manipulations.

Note that at high-SNRs, the sum rate converges to

$$
\begin{aligned}
& R^{\infty}=L N_{t} \log _{2}\left(\frac{\rho}{L N_{t}}\right)+\frac{L N_{t}}{\ln 2} \psi\left(N_{r}-L N_{t}+1\right) \\
& -\sum_{m=1}^{L N_{t}} \log _{2}\left(\sigma_{m}\right)+N_{t} \sum_{m=1}^{L}\left(\frac{\psi\left(k_{m}\right)}{\ln 2}+\log _{2}\left(\frac{\Omega_{m}}{D_{m}^{v}}\right)\right) .
\end{aligned}
$$

where $\psi(x)$ is Euler's digamma function [11, Eq. (8.360.1)]. The above expression is quite intuitive since it indicates that at high SNRs the effects of small and large-scale fading are decoupled. We also validate the diminishing effects of spatial correlation on the sum rate since, due to Hadamard's inequality, $1 \leq \operatorname{det}\left(\mathbf{R}_{t}^{-1}\right) \leq \prod_{m=1}^{L N_{t}} \sigma_{m}$, with equality for $\mathbf{R}_{t}=\mathbf{I}_{L N_{t}}$. Clearly, larger values of $k_{m}, \Omega_{m}$ diminish the effects of shadowing thereby delivering higher sum rate while longer terminal distances, $D_{m}$, lead to stronger path-loss attenuation.

\subsection{Low-SNR analysis}

It has been theoretically shown that the low-SNR sum rate can be more effectively explored in terms of the normalized transmit energy per information bit $E_{b} / N_{0}$ rather than persymbol SNR, or $[12,13]$

$$
R\left(\frac{E_{b}}{N_{0}}\right) \approx \mathcal{S}_{0} \log _{2}\left(\frac{\frac{E_{b}}{N_{0}}}{\frac{E_{b}}{N_{0} \min }}\right)
$$

where $\frac{E_{b}}{N_{0}}$ min and $\mathcal{S}_{0}$ correspond to the "minimum normalized energy per information bit required to convey any positive rate reliably" and the wideband slope, respectively. 
Corollary 1 The minimum energy per information bit and the wideband slope of $Z F$ detectors over correlated K MIMO channels are respectively

$$
\begin{aligned}
\frac{E_{b}}{N_{0 \text { min }}} & =\frac{L N_{t} \ln 2}{\left(N_{r}-L N_{t}+1\right)}\left(\sum_{m=1}^{L N_{t}} \frac{k_{m} \Omega_{m}}{\sigma_{m} D_{m}^{v}}\right)^{-1} \\
\mathcal{S}_{0} & =\frac{2\left(N_{r}-L N_{t}+1\right)}{\left(N_{r}-L N_{t}+2\right)} \frac{\left(\sum_{m=1}^{L N_{t}} \frac{k_{m} \Omega_{m}}{\sigma_{m} D_{m}^{v}}\right)^{2}}{\sum_{m=1}^{L N_{t}} k_{m}\left(k_{m}+1\right)\left(\frac{\Omega_{m}}{\sigma_{m} D_{m}^{v}}\right)^{2}} .
\end{aligned}
$$

Proof: From [13], we have that $\frac{E_{b}}{N_{0} \text { min }}$ and $\mathcal{S}_{0}$ are respectively

$$
\frac{E_{b}}{N_{0} \text { min }} \triangleq \lim _{\rho \rightarrow 0} \frac{\rho}{R(\rho)}=\frac{1}{\dot{R}(0)} \text { and } \mathcal{S}_{0} \triangleq-\frac{2 \ln 2[\dot{R}(0)]^{2}}{\ddot{R}(0)}
$$

where $\dot{R}(\cdot)$ and $\ddot{R}(\cdot)$ denote the first and second-order derivatives of the sum rate (6) over the $\operatorname{SNR} \rho$. The desired results follow easily after appropriate simplifications.

For i.i.d. Rayleigh fading, it can be shown that $\frac{E_{b}}{N_{0} \min }=$ $\ln 2 /\left(N_{r}-N_{t}+1\right)$ and $\mathcal{S}_{0}=2 N_{t}\left(N_{r}-N_{t}+1\right) /\left(N_{r}-N_{t}+2\right)$, which are respectively identical with [15, Eq. (25)] and [15, Eq. (26)]. In the specific case of $N_{t}=1$, the slope particularizes to $\mathcal{S}_{0}=2 N_{r} /\left(N_{r}+1\right)$ which coincides with the associated results for both MMSE [14, Eq. (52)], [15, Eq. (24)] and optimal receivers [12, Eq. (16)], thereby validating that at low SNRs, ZF detection becomes optimal for a single transmit antenna since all $N_{r}$ degrees of freedom are devoted to the recovery of the corresponding multiplexed stream. All these results further demonstrate that for a fixed $N_{r}$, increasing $N_{t}$ may improve the wideband slope, but also increases the minimum energy per bit due to the additional power required to cancel out the extra interferers.

\subsection{Tight bounds on the sum rate}

Since the expression in Theorem 1 is given via a Meijer- $G$ function, which is a general function with a hard practical interpretation, it is important to obtain tight upper and lower closed-form bounds on the achievable sum rate.

Theorem 2 The achievable sum rate of $Z F$ detectors over correlated K MIMO channels in (6) is upper bounded by

$$
R_{U}=\sum_{m=1}^{L N_{t}} \log _{2}\left(1+\frac{\rho\left(N_{r}-L N_{t}+1\right)}{L N_{t}} \frac{k_{m} \Omega_{m}}{\sigma_{m} D_{m}^{v}}\right) .
$$

Proof: The proof follows by applying Jensen's inequality on (6) along with the methodology of [16, Theorem 4].
Corollary 2 In the high-SNR regime, the upper bound in (10) becomes

$$
\begin{aligned}
R_{U}^{\infty} & =L N_{t} \log _{2}\left(\frac{\rho}{L N_{t}}\right)+L N_{t} \log _{2}\left(N_{r}-L N_{t}+1\right) \\
& -\sum_{m=1}^{L N_{t}} \log _{2}\left(\sigma_{m}\right)+N_{t} \sum_{m=1}^{L} \log _{2}\left(\frac{k_{m} \Omega_{m}}{D_{m}^{v}}\right) .
\end{aligned}
$$

Interestingly, the upper bound becomes tighter for higher $N_{r}, k_{m}$. In the limit, $N_{r}, k_{m} \rightarrow \infty$, the bound becomes exact since $\psi(x) \approx \ln (x)$ if $x \rightarrow \infty$. On a similar basis, we can now propose the following new lower bound on the sum rate, which becomes exact at high-SNRs.

Theorem 3 The achievable sum rate of ZF detectors over correlated K MIMO channels in (6) is lower bounded by

$$
\begin{aligned}
R_{L} & =\sum_{m=1}^{L N_{t}} \log _{2}\left(1+\frac{\rho}{L N_{t}} \exp \left(\psi\left(N_{r}-L N_{t}+1\right)\right.\right. \\
& \left.\left.+\psi\left(k_{m}\right)+\ln \left(\frac{\Omega_{m}}{\sigma_{m} D_{m}^{v}}\right)\right)\right) .
\end{aligned}
$$

Proof: The proof relies on the generic bounding technique of [2, Theorem 1] which is omitted for the sake of brevity. A detailed proof is given in a journal version of this paper [16].

\section{NUMERICAL RESULTS}

We first generate 20,000 random realizations of the small and large-scale fading matrices $\mathbf{H}$ and $\boldsymbol{\Xi}$ according to (2), and thereafter obtain the simulated sum rate via (6). The transmit correlation matrix is constructed as $\mathbf{R}_{t}=\operatorname{diag}\left\{\mathbf{R}_{t, m}\right\}_{m=1}^{L}$ where $\mathbf{R}_{t, m}$ is the correlation matrix between the antennas of the $m$-th terminal. The entries of the latter are modeled via the common exponential correlation model $\left\{\mathbf{R}_{t, m}\right\}_{i, j}=\rho_{t, m}^{|i-j|}$ with $\rho_{t, m} \in[0,1)$ being the transmit correlation coefficient.

In Fig. 1, the achievable sum rate is plotted vs the SNR, $\rho$. For the sake of simplicity, we have set $k_{m}=1, \Omega_{m}=$ $2, \rho_{t, m}=0.3, \forall m=1, \ldots, L N_{t}$ while we consider different MIMO configurations by keeping $N_{t}=2$ and increasing only $N_{r}$. The outputs of a Monte-Carlo simulator are compared with the exact analytical expression of Theorem 1 and the upper/lower bounds of (10) and (12), respectively. It is easily observed that the match between theory and simulation is excellent in all cases under consideration, thereby validating the correctness of the proposed analytical expressions. The graph indicates that adding more receive antennas significantly stabilizes the MIMO link by improving the receive diversity and reducing the noise enhancement effect. This is consistent with the results in [9]. Further, a higher $N_{r}$ makes both bounds tighter although the upper bound is in general less tight due to the loose nature of Jensen's inequality. As previously mentioned, at high SNRs the lower bound becomes exact. 


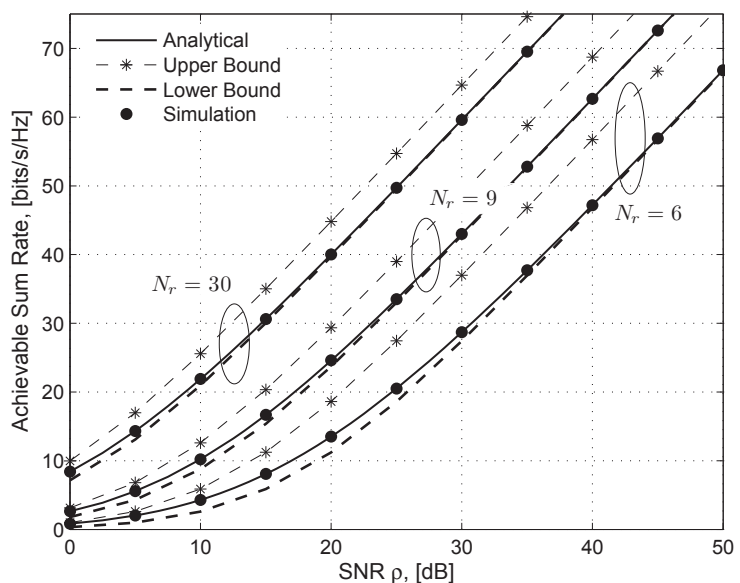

Fig. 1. Simulated sum rate, analytical expression and upper/lower bounds against the $\operatorname{SNR}\left(L=3, v=4, D_{1}=1000\right.$ $\left.\mathrm{m}, D_{2}=1500 \mathrm{~m}, D_{3}=2000 \mathrm{~m}\right)$.

In Fig. 2, the analytical and simulated low-SNR sum rate are depicted against the transmit energy per bit $E_{b} / N_{0}$ (Corollary 1). For illustration purposes, we consider only smallscale fading (both correlated and i.i.d. Rayleigh fading). It can be observed that increasing $N_{t}$ increases the minimum energy per bit and so does the correlation coefficient $\rho_{t}$. The wideband slope is enhanced with a higher $N_{t}$ and seems to remain relatively unaffected by spatial correlation. For all scenarios, it turns out that the linear approximations are quite accurate over a moderate range of $E_{b} / N_{0}$ values.

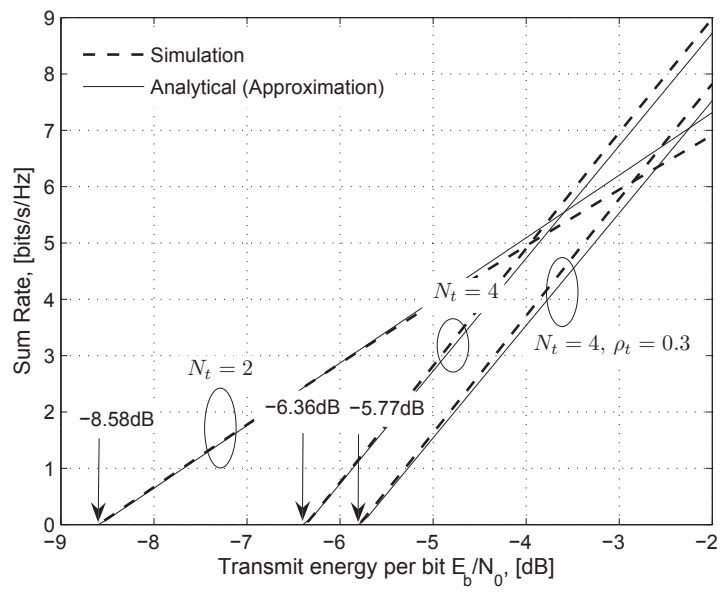

Fig. 2. Low-SNR simulated sum and analytical linear approximation against the transmit energy per bit $\left(N_{r}=6\right)$.

\section{CONCLUSION}

The main focus of this paper was on the sum rate characterization of ZF detectors over correlated $K$ MIMO channels.
To this end, a novel analytical formula for the exact maximum achievable sum rate was provided along with a tractable affine expansion in the asymptotically low-SNR regime. Simple closed-form upper and lower bounds were also provided, which were demonstrated to remain tight across the entire SNR range. We finally explored via numerical simulations, the implications of the model parameters on the performance of ZF detectors over composite $K$ fading MIMO channels.

\section{REFERENCES}

[1] I. E. Telatar, "Capacity of multi-antenna Gaussian channels," Europ. Trans. Telecommun., vol. 10, no. 6, pp. 585-595, Nov./Dec. 1999.

[2] Ö. Oyman, R. Nabar, H. Bölcskei, and A. Paulraj, "Characterizing the statistical properties of mutual information in MIMO channels," IEEE Trans. Signal Process., vol. 51, no. 11, pp. 2782-2795, Nov. 2003.

[3] H. Zhang and H. Dai, "On the capacity of distributed MIMO systems," in Proc. Conf. Inform. Sciences and Systems (CISS), Princeton University, Princeton, NJ, Mar. 2004.

[4] H. Dai, "Distributed versus co-located MIMO systems with correlated fading and shadowing," in Proc. IEEE Int. Conf. Acoustics Speech Signal Proc. (ICASSP), Toulouse, France, May 2006.

[5] H. Dai, H. Zhang, and Q. Zhou, "Some analysis in distributed MIMO systems," J. Commun., vol. 2, no. 3, pp. 43-50, May 2007.

[6] A. Abdi and M. Kaveh, " $K$ distribution: An appropriate substitute for Rayleigh-lognormal distribution in fading-shadowing wireless channels," IEE Electron. Lett., vol. 34, no. 9, pp. 851-852, Apr. 1998.

[7] - "Comparison of DPSK and MSK bit error rates for $K$ and Rayleighlognormal fading distributions," IEEE Commun. Lett., vol. 4, no. 4, pp. 122-124, Apr. 2000.

[8] M. Park, C. B. Chae, and R. W. Heath, Jr., "Ergodic capacity of spatial multiplexing MIMO channels with log-normal shadowing and Rayleigh fading" in Proc. IEEE Int. Symp. Personal Indoor Mobile Radio Commun. (PIMRC), Athens, Greece, Sept. 2007.

[9] A. Paulraj, R. Nabar, and D. A. Gore, Introduction to Space-Time Wireless Communications, Cambridge University Press, May 2003.

[10] D. A. Gore, R. W. Heath, Jr., and A. Paulraj, "Transmit selection in spatial multiplexing systems," IEEE Commun. Lett., vol. 6, no. 11, pp. 491-493, Nov. 2002.

[11] I. S. Gradshteyn and I. M. Ryzhik, Table of Integrals, Series, and Products, Seventh Ed. Academic Press, San Diego, 2007.

[12] A. Lozano, A. M. Tulino, and S. Verdú, "Multiple-antenna capacity in the low-power regime," IEEE Trans. Inf. Theory, vol. 49, no. 10, pp. 2527-2544, Oct. 2003

[13] S. Verdú, "Spectral efficiency in the wideband regime," IEEE Trans. Inf. Theory, vol. 48, no. 6, pp. 1319-1343, June 2001.

[14] M. R. McKay, I. B. Collings, and A. M. Tulino, "Achievable sum rate of MIMO MMSE receivers: A general analytic framework," IEEE Trans. Inf. Theory, vol. 56, no. 1, pp 396-410, Jan. 2010.

[15] R. H. Y. Louie, M. R. McKay, and I. B. Collings, "Sum capacity of MIMO multiuser scheduling with linear receivers," IEEE Trans. Commun., vol. 57, no. 11, pp. 3500-3510, Nov. 2009.

[16] M. Matthaiou, N. D. Chatzidiamantis, G. K. Karagiannidis, and J. A. Nossek, "ZF detectors over correlated $K$ fading MIMO channels," in press IEEE Trans. Commun., 2011 